\title{
Friction Dynamics of Wood Coated with Vegetable Oil
}

\author{
Yoshimune Nonomura ${ }^{1 *}$, Momoka Sano ${ }^{1}$, Ryota Sekine ${ }^{1}$, and Yoshiyuki Daikoku ${ }^{2}$ \\ ${ }^{1}$ Department of Biochemical Engineering, Graduate School of Science and Engineering, Yamagata University, 4-3-16 Jonan, Yonezawa \\ 992-8510, JAPAN \\ ${ }^{2}$ Osmo \& Edel Japan Co., Ltd., 748-6 Kamiarakawa, Yokawa-cho, Miki-city 673-1111, JAPAN
}

\begin{abstract}
Surface treatment of wood surface is an effective method to improve the physical properties. The friction dynamics of wood coated with vegetable oil were evaluated and compared to wood treated with polyurethane and untreated wood. The kinetic friction coefficient, $\mu_{k}$, was $0.39 \pm 0.01$, which was smaller than the values for polyurethane-treated wood and untreated oak. The effect of the surface treatment was also observed in the dependence of velocity on the friction coefficient. The friction profile of the wood surface treated with vegetable oil was similar to that of untreated wood, and the friction coefficient was nearly constant, except in the static friction region of sliding out. These results suggest that wood treated with vegetable oil is suitable for inducing a smooth feel.
\end{abstract}

Key words: vegetable oil, polyurethane, wood, coating, friction

\section{Introduction}

Surface treatment with vegetable oils is an effective method to improve the physical properties of wood. Treatment with palm oil, soybean oil, or slack wax under high heat conditions improves the water absorption, moisture absorption, and shrinkage resistance of wood ${ }^{1)}$. Heat treatment of Pinus radiata wood in flaxseed oil resulted in a more uniform and darker wood color and improved fungal resistance $^{2)}$. Oil heat treatment significantly increased the decay resistance of aspen wood(Populus tremula 1.) to white and brown rot fungi ${ }^{3)}$. Vegetable oil is retained on the surface by hydrogen bonds between the hydroxyl groups and the ester functional groups in wood carbohydrates ${ }^{4)}$. Recently, derivatives of triacylglycerol contained in vegetable oils have attracted attention as wood surface modifiers ${ }^{5,6)}$.

Friction and wear properties of wood are important factors that affect its durability and tactile feel. McKenzie et al. reported that for most dry trees, the static friction coefficient is 0.65 and the kinetic friction coefficient at $4 \mathrm{~m}$ $\mathrm{s}^{-1}$ is approximately 0.4 , while for wet trees, the static friction coefficient and the kinetic friction coefficient are 0.65 and 0.15 , respectively ${ }^{7}$. Klamecki evaluated the variation of the friction coefficient with normal load and tool surface finish for low-speed orthogonal cutting of white fir and showed that the friction coefficient is independent of tool roughness in the range of roughness of well-finished cutting tools ${ }^{8)}$.

In conventional friction evaluation methods, the friction force when a contact probe slides at a constant velocity has been evaluated. However, under conditions where the velocity is constantly changing, some interesting information on friction dynamics can be obtained from a single evaluation result, such as the friction force on a small timescale at the beginning of motion, the velocity dependence of the friction force over a wide range, and possible hysteresis in these values. Therefore, we developed a sinusoidal motion friction evaluation system to evaluate the friction occurring under conditions of nonlinear motion ${ }^{9,10)}$. With this system, we can obtain the delay time $\delta$ between the motion of the contact probe at the start of motion and the mechanical response, as well as the viscosity coefficient $C$, which is obtained from the profile of the friction force in the kinetic friction process and can quantify the velocity dependence over several scales. This system is also useful for analyzing the mechanisms of tactile and food sensations. In general, when we touch objects or eat food, our fingers and tongue move in nonlinear motion with constantly changing velocity. In previous studies, we have shown the mechanism of the tactile feel of cosmetic sponges, powders, leather, and cloth using a sinusoidal motion friction evaluation system ${ }^{11-14)}$.

In this study, the surface topography and optical and thermal properties of wood coated with vegetable oil(1)

\footnotetext{
* Correspondence to: Yoshimune Nonomura, Department of Biochemical Engineering, Graduate School of Science and Engineering, Yamagata University, 4-3-16 Jonan, Yonezawa 992-8510, JAPAN

E-mail: nonoy@yz.yamagata-u.ac.jp ORCID ID: https://orcid.org/0000-0003-0461-124X

Accepted August 24, 2021 (received for review June 22, 2021)

Journal of Oleo Science ISSN 1345-8957 print / ISSN 1347-3352 online

http://www.jstage.jst.go.jp/browse/jos/ http://mc.manusriptcentral.com/jjocs

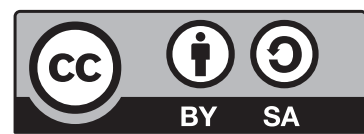




\section{Y. Nonomura, M. Sano, R. Sekine et al.}

were evaluated, and the friction dynamics were analyzed using a sinusoidal motion friction evaluation system. Similar evaluations were performed on wood coated with polyurethane (2) and untreated wood (3) to compare their characteristics to the surface treatment with vegetable oil.

\section{Experimental}

\subsection{Materials}

Wood coated with vegetable oil 1 was prepared as follows. An oak board was sanded with 400 grit sandpaper and then painted with Osmo color wood wax (Osmo \& Edel Co., Ltd., Miki City, Hyogo Prefecture, Japan) using a spray gun. After drying, the coated surface was sanded smooth with 400 grit sandpaper and then coated again with Osmo color wood wax. The ingredients of Osmo color wood wax are as follows: oil (40 wt\%; sunflower oil, soybean oil, and thistle oil), wax (10 wt\%; carnauba wax and candelilla wax), solvent (40 wt\%; dearomatized white mineral oil), pigment, earth, and additives. Wood coated with polyurethane 2 was prepared as follows. An oak board was sanded with 180 grit sandpaper and then painted with polyurethane resin wood sealer (Otani Paint, Osaka, Japan) using a spray gun. After drying, the surface of the wood was sanded smooth with 400 grit sandpaper. The wood surface was then painted with a polyurethane resin wood sealer / lacquer thinner mixture (1:1 by weight), and after drying, the coated surface was polished smooth with 400 grit sandpaper. Finally, the wood surface was painted again with the paint/thinner mixture using a spray gun. The untreated wood 3 was made of oak.

\subsection{Evaluation method}

Wood surfaces were photographed using a DZ-D100 Dermo Camera (Yamagata Casio Co., Ltd., Yamagata, Japan). Arithmetic mean roughness value $R_{a}$ and 10-point average roughness value $R_{z}$ were measured using a LEXT OLS4000 3D Laser Measuring Microscope (Olympus Corporation, Tokyo, Japan). Colorimetric $L^{*} a^{*} b^{*}$ values were measured using a CM-700d Spectrophotometer (Konica Minolta, Tokyo, Japan). Gloss was measured using a handheld IG-331 Gloss Checker(Horiba Co., Ltd., Kyoto, Japan). The angle of incidence was $20^{\circ}$, and the light source was an LED light (wavelength $=890 \mathrm{~nm}$ ). The heat flux $q$-max was measured using a KES-F7 Thermo Labo (Kato Tech Co., Ltd., Kyoto, Japan). The measurement was carried out in an environment where the temperature and humidity were $20^{\circ} \mathrm{C} \pm 2{ }^{\circ} \mathrm{C}$ and $50 \% \pm 5 \%$, respectively. The abrasion resistance of the wood was evaluated based on the method described in JIS K 5600-5-10 using an NUS-IS03 abrasion resistance tester (Suga Testing Machine Co., Ltd., Tokyo, Japan). The evaluation conditions were as follows: Abrasive material $=$ silicon carbide grinding material $(\mathrm{C})$ (grain size
$\mathrm{P} 1200), \operatorname{load}=2.94 \pm 0.05 \mathrm{~N}$, reciprocating frequency $=400$ times. The contact angles of water on wood 1-3 were estimated using a DM-501 contact angle meter (Kyowa Interface Science, Tokyo, Japan) based on a sessile drop-measuring method. The contact angle was calculated by a half angle method.

Figure 1 shows photographs and a schematic of the friction evaluation system ${ }^{9)}$. A scotch yoke mechanism, which converts the linear motion of a slider into rotational motion, was used to model the simple harmonic motion of the location of the piston versus time. The brushless DC motor has a rotational speed of 0.01 to $2.1 \mathrm{rad} \mathrm{s}^{-1}$ and a measurement distance of \pm 2.5 to $20 \mathrm{~mm}$. The moving distance was measured by a CD22-100VM122 laser displacement meter(Optex-A, Tokyo, Japan) mounted on the side of the drive motor. The mechanical stimulus on the bottom side is sensed by two load cells to measure the stress in the tangential and normal directions. The measurement ranges of $F_{x}$ and $F_{z}$ were 0.06 to $9.9 \mathrm{~N}$ and 0.06 to $9.8 \mathrm{~N}$, respectively. A model of a finger was made from polyurethane resin and was used as a contact probe. On the surface of the finger model, grooves with a depth of $0.15 \mathrm{~mm}$ were
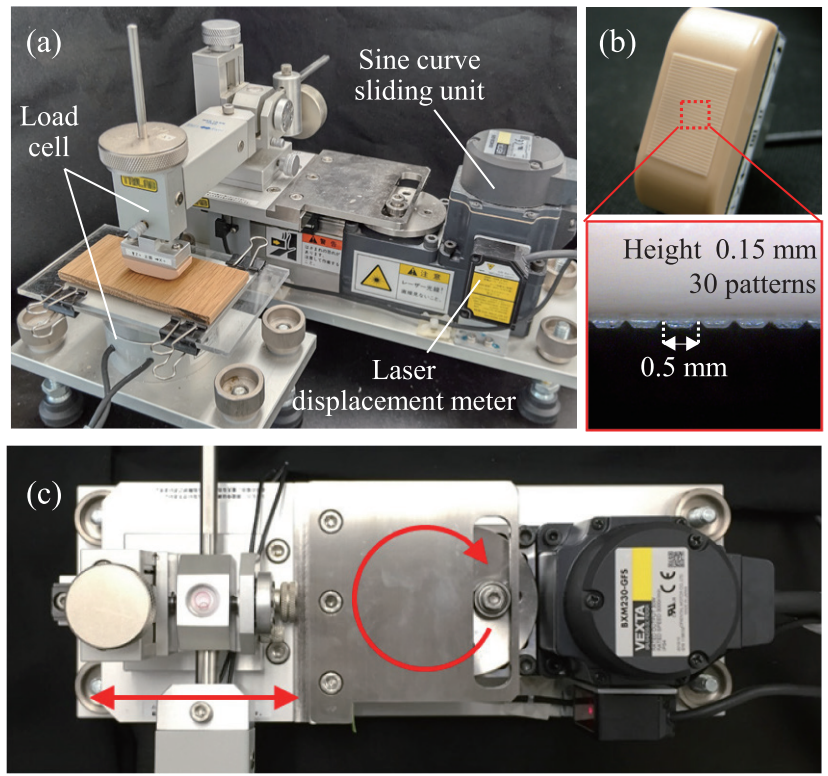

(d)

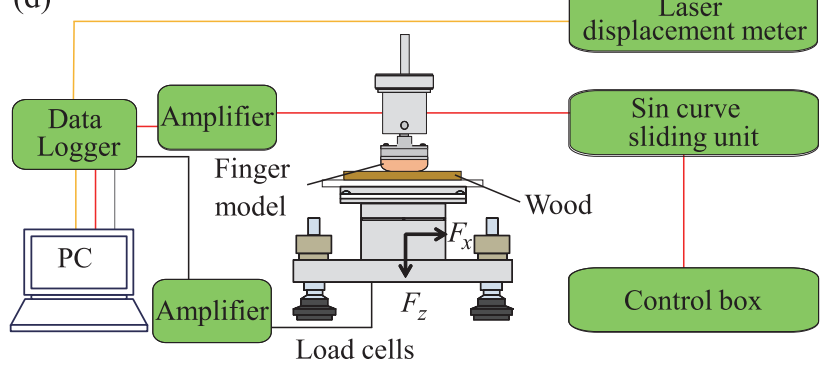

Fig. 1 Friction evaluation system. (a) Overall view (b) Finger model (c) Drive unit (d) Schematic diagram. 
engraved at intervals of $0.5 \mathrm{~mm}^{15)}$. The velocity $V$ during sine motion is expressed by equation(1) using the amplitude $A$, angular velocity $\omega$, and time $T$.

$$
V=\lfloor A \mid \omega \cos \omega T
$$

The $A, \omega$, and vertical loads were $14.5 \mathrm{~mm}, 2.1 \mathrm{rad} \mathrm{s}^{-1}$, and $0.98 \mathrm{~N}$, respectively. The maximum sliding velocity was $31 \mathrm{~mm} \mathrm{~s}^{-1}$. There were eleven round trips.

\section{Results and Discussion}

The surface morphology and physical properties of wood 1 are shown in Table 1. Laser micrographs show that the surface texture of wood 1 had an arithmetic mean roughness $R_{a}$ of $0.93 \pm 0.29 \mu \mathrm{m}$ and a ten-point mean roughness $R_{z}$ of $4.95 \pm 1.57 \mu \mathrm{m}$. In comparison, the $R_{a}$ and $R_{z}$ of wood 3 were $4.40 \pm 3.38$ and $21.99 \pm 13.45 \mu \mathrm{m}$, respectively, where the difference indicates that the surface of wood 1 was covered by oil and wax and slightly smoothed. However, the degree of smoothing was less than that of the surface of the wood 2; its surface texture was quite different from that of woods 1 and 3 , with $R_{a}$ and $R_{z}$ values of $0.33 \pm 0.11$ and $1.24 \pm 0.37 \mu \mathrm{m}$, respectively. These data show that the polymeric paint almost completely filled and smoothed the concave areas of the wood surface. We estimated the thickness of the film based on laser microscope images. In the case of wood 1, wax or oil was adhered to the surface of the wood, because the surface properties were different from those of wood 3 . However, the thickness of the coated phase could not be evaluated because of the large surface roughness. On the other hand, in the case of wood 2, the reflected light at both the surface of the coated phase and the wood substrate was observed. The gap between the layers on both sides was about $40 \mu \mathrm{m}$, which corresponds to the thickness of the polyurethane film.

The change in surface composition and morphology

Table 1 Physical properties of woods treated with vegetable oil(1), polyurethane(2), and untreated $\operatorname{wood}(3)$.

\begin{tabular}{|c|c|c|c|}
\hline & $\begin{array}{c}\underline{1} \\
\text { Vegetable oil }\end{array}$ & $\begin{array}{c}\underline{\mathbf{2}} \\
\text { Polyurethane }\end{array}$ & $\begin{array}{c}\underline{\mathbf{3}} \\
\text { Untreated }\end{array}$ \\
\hline \multicolumn{4}{|l|}{ Appearance } \\
\hline \multicolumn{4}{|l|}{ Microscopic image } \\
\hline \multicolumn{4}{|l|}{ Surface roughness } \\
\hline$R_{a} / \mu \mathrm{m}$ & $0.93 \pm 0.29$ & $0.33 \pm 0.11$ & $4.40 \pm 3.38$ \\
\hline$R_{z} / \mu \mathrm{m}$ & $4.95 \pm 1.57$ & $1.24 \pm 0.37$ & $21.99 \pm 13.45$ \\
\hline \multicolumn{4}{|l|}{ Color measurement } \\
\hline$L^{*}$ & 53.56 & 54.47 & 61.18 \\
\hline$a^{*}$ & 10.06 & 8.6 & 6.98 \\
\hline$b^{*}$ & 24.69 & 22.18 & 20.83 \\
\hline Gloss & $5.7 \pm 0.6$ & $77.0 \pm 0.0$ & $1.0 \pm 0.3$ \\
\hline \multicolumn{4}{|l|}{ Thermal property } \\
\hline$q-\max / \mathrm{W} \mathrm{cm}-2$ & $0.252 \pm 0.009$ & $0.218 \pm 0.018$ & $0.150 \pm 0.006$ \\
\hline \multicolumn{4}{|l|}{ Wear resistance } \\
\hline Abrasion loss / mg & $9 \pm 1$ & $10 \pm 1$ & $5 \pm 1$ \\
\hline \multicolumn{4}{|l|}{ Wettability (Water) } \\
\hline Contact angle $/^{\circ}$ & $79.6 \pm 0.6$ & $65.6 \pm 0.9$ & $38.3 \pm 5.0$ \\
\hline
\end{tabular}


caused by the polymeric paint had a significant effect on the optical and thermophysical properties of the wood. First, the gloss value of untreated wood 3 was $1.0 \pm 0.3$, while those of woods 1 and 2 were $5.7 \pm 0.6$ and $77.0 \pm 0.0$, respectively. In other words, the gloss of wood 2 with a smooth surface covered by polyurethane was noticeably higher, while that of wood 1 was only slightly higher than that of untreated wood 3 . In addition, for the colorimetric data, the surface treatment significantly reduced the $L^{*}$ value and increased the $a^{*}$ and $b^{*}$ values, because the smoothing of the surface suppressed the scattering of light. The $q$-max, which indicates the ease of heat transfer from the wood, also increased with the surface treatment. One possible mechanism for this change is that the area contacted by the contact probe increased and led to greater heat transfer because of the smoother surface. In addition, the effect of surface treatment on wear resistance and wettability were studied. The wear loss of woods 1 to 3 , measured according to the JIS method, were $9 \pm 1,10 \pm 1$, and $5 \pm 1 \mathrm{mg}$, respectively. The wear loss of wood 1 was almost the same as that of wood 2 . These results suggest that the surface treatment with vegetable oil has the same level of wear resistance as the surface treatment with polyurethane. Untreated wood surfaces also showed high wear resistance since oak is relatively hard ${ }^{16)}$. In addition, the contact angle of a water droplet on the wood 1-3 was 79.6 $\pm 0.6,65.6 \pm 0.9,38.3 \pm 5.0$ degree, respectively. These data suggest that treatment with vegetable oil improves the water repellency of the wood.

Our present evaluation shows that surface treatment with vegetable oil also has a significant effect on friction dynamics. When wood 1 was rubbed with a contact probe at a velocity of $2.1 \mathrm{rad} \mathrm{s}^{-1}$ and a normal load of $0.98 \mathrm{~N}$, the friction coefficient increased with acceleration and reached the static friction coefficient $\mu_{s}$ of 0.52 where the friction coefficient is the ratio of the friction force to the vertical force (Fig. 2). Under sinusoidal motion, a time difference $\Delta T$ was observed between the start of the probe's movement and the response of the friction force; the delay time $\delta$ was 0.013 , which is the value normalized by the time $T_{0}$. $T_{0}$ is the time required for the contact probe to make one round trip. Thereafter, the friction coefficient decreased and the average $\mu_{k}$ during the kinetic friction process was $0.39 \pm 0.01$. At $T / T_{0}=0.5$, the velocity of the contact probe was zero and the direction of motion was reversed. The friction coefficients in the positive and negative velocity regions represent the data for the outward and homeward directions, respectively. A weak oscillation phenomenon was observed before and after the reverse. This phenomenon is often observed in sinusoidal motion friction evaluation, because the stick-slip phenomenon occurs under conditions where the friction velocity and vertical load are moderate for the oscillation phenomenon ${ }^{17}$. In this case, these conditions were satisfied at the velocity was about 10

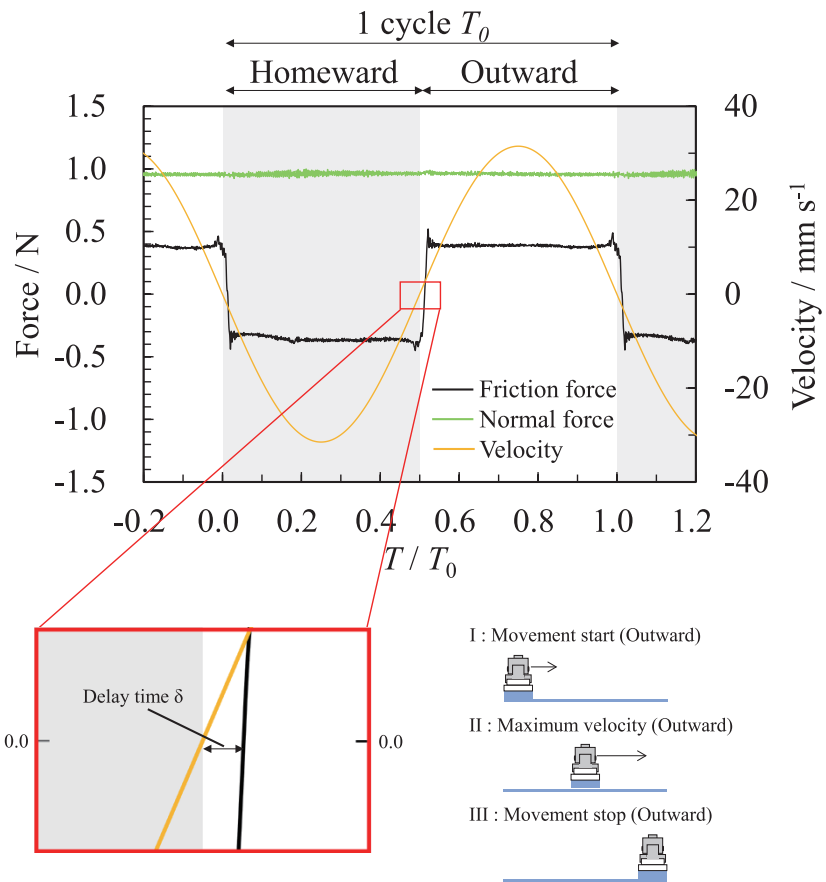

Fig. 2 Temporal profiles of the friction force(black line), the velocity (yellow line), and the normal force (green line) on the wood surface treated with vegetable oil(1) at $\omega=2.1 \mathrm{rad} \mathrm{s}^{-1}$ and $W=0.98 \mathrm{~N}$.

$\mathrm{mm} \mathrm{s}^{-1}$.

The friction parameters of wood 1 showed the lowest values of $\mu_{s}, \mu_{k}$, and $\delta$ for all three woods (Fig. 3). The parameters $\mu_{s}$ and $\mu_{k}$ of wood 1 was slightly smaller than those of wood 3, but $\delta$ does not appear to be significantly different. The lower friction of wood 1 compared to wood 3 was expected because vegetable oil has lower surface energy than wood, and thus acts as a lubricant between the contact probe and the wood. While the surface tension of common wood is about $35 \mathrm{mNm}^{-1}$, the surface tensions of vegetable oil and mineral oil, the main ingredients of Osmo color wood wax, are 30 to $34 \mathrm{mNm}^{-1}$ and $28 \mathrm{mNm}^{-1}$, respectively ${ }^{18-20)}$. The $\mu_{s}$ and $\mu_{k}$ of wood 2 were $1.00 \pm 0.08$ and $0.75 \pm 0.03$, respectively, which were significantly larger than those of woods 1 and 3 . The large real contact area between the contact probe and wood surface may have increased the friction force and delay time; the parameters $R_{a}$ and $R_{z}$ are $0.33 \pm 0.11$ and $1.24 \pm 0.37 \mu \mathrm{m}$, which are smaller than those of woods 1 and 3 (Table 1 ). In the present system, the contact state cannot be directly observed. However, Nishi et al. reported that the ratio of the real contact area against the apparent contact area was about $1 \%$ when the probe was contacted with a flat glass at vertical load $=0.98 \mathrm{~N}^{21}$. According to Hertz's contact theory, the contact area becomes smaller if the contact surface is rougher ${ }^{22)}$. These findings suggest that the contact area between wood 2 and the contact probe is larger than that of wood 1 or 3 . 


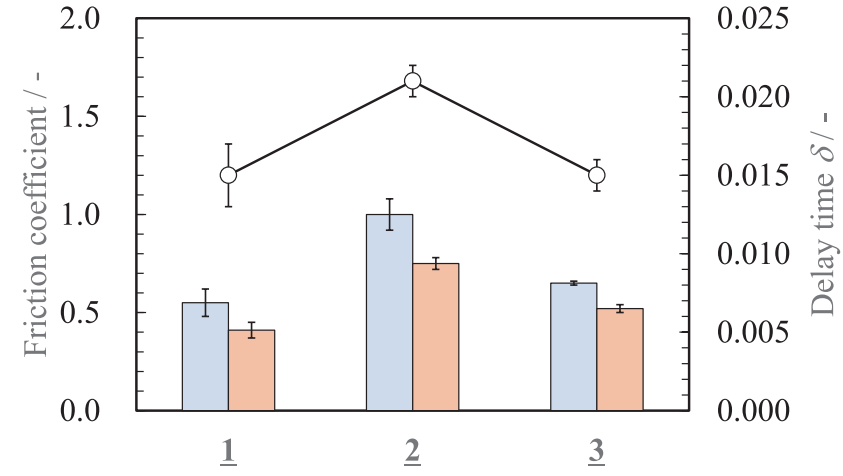

Fig. 3 Friction parameters of woods treated with vegetable oil (1) and polyurethane (2), and of untreated $\operatorname{wood}(3)$. Static friction coefficient $\mu_{s}$ $(\square)$, kinetic friction coefficient $\mu_{k}(\square)$, and delay time $\delta(\bigcirc)$.

Furthermore, the effect of the surface treatment was observed in the velocity-dependent profile of the friction coefficient (Fig. 4). The friction profile of wood 1 was similar to that of wood 3 , in which the friction coefficient was almost constant except in the static friction region of sliding out. Previously, we proposed a friction model for viscoelastic material surfaces to consider these characteristic phenomena ${ }^{9,10)}$. Based on this framework, the weak velocity dependence of the friction coefficient indicates that the elastic factor is dominant among the viscous and elastic responses. On the other hand, in the case of wood 2, an increase in the friction coefficient was observed in the deceleration region of the dynamic friction process; the friction coefficient in the low-velocity region $(-8$ to +8 $\mathrm{mm} \mathrm{s}^{-1}$ ) was about 0.5 higher than that in the high velocity region. This suggests that reattachment occurred between the polyurethane surface and the contact probe in this deceleration region.

These results suggest that the surface properties of wood treated with vegetable oil are suitable to induce a smooth feel. Our previous studies showed that the smoothness of wood is enhanced when the coefficient of friction and the variation in the dynamic friction process are small and the surface has micron-order irregularities ${ }^{12,13)}$. The frictional properties and surface morphology of wood 1 meet these requirements, suggesting that treatment with vegetable oil is a useful method for the improvement of the tactile feel of wood.

\section{Conclusions}

In this study, we found that the surface of wood coated with vegetable oil has a low coefficient of friction and is suitable to induce a smooth feel. This suggests that these materials have excellent properties as naturally occurring coating agents for wood surfaces. The effects of several
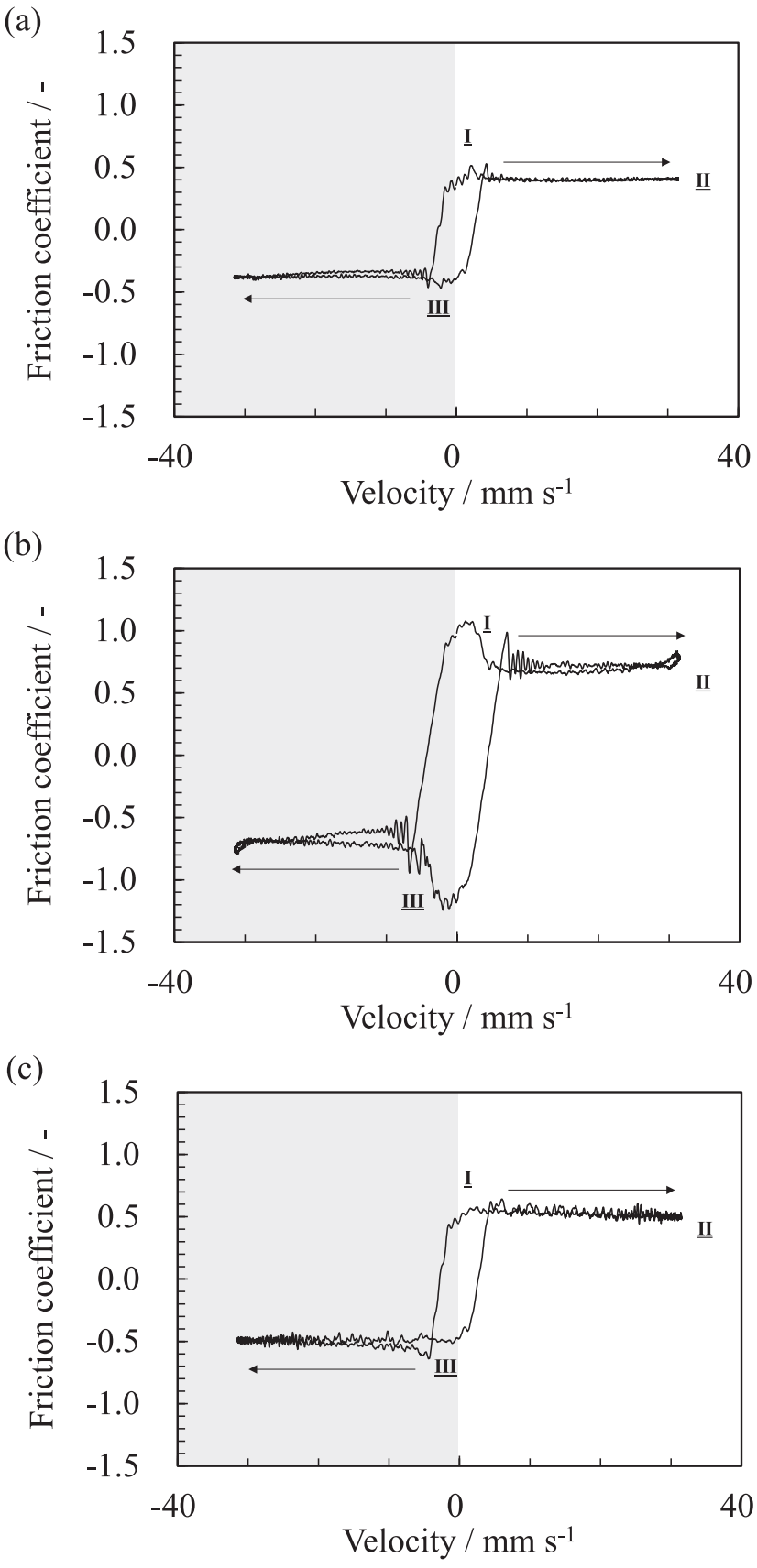

Fig. 4 Friction profiles of woods treated with (a) vegetable oil (1) and (b) polyurethane (2), and (c) of untreated $\operatorname{wood}(3)$.

factors, e.g., wood lot, wood grain, and coating uniformity, should be studied in order to determine the more suitable conditions.

\section{Acknowledgment}

This study was partly supported by the Yamagata University YU-COE (S) program and Osmo \& Edel Co. Ltd. 


\section{References}

1) Wang, J.Y.; Cooper, P.A. Effect of oil type, temperature and time on moisture properties of hot oil-treated wood. Holz. Als. Roh- und Werkstoff. 63, 417-422 (2005).

2) Dubey, M.K.; Pang, S.; Walker, J. Changes in chemistry, color, dimensional stability and fungal resistance of Pinus radiata D. Don wood with oil heat-treatment. Holzforschung 66, 49-57 (2012).

3) Bazyar, B. Decay resistance and physical properties of oil heat treated aspen wood. BioResources 7, 696-705 (2012).

4) Lee, S.H.; Ashaari, Z.; Lum, W.C.; Halip, J.A.; Ang, A.F.; Tan, L.P.; Chin, K.L.; Tahir, P.M. Thermal treatment of wood using vegetable oils: A review. Const. Build. Materials 181, 408-419(2018).

5) Wuzella, G.; Mahendran, A.R.; Müller, U.; Kandelbauer, A.; Teischinger, A. Photocrosslinking of an acrylated epoxidized linseed oil: Kinetics and its application for optimized wood coatings. J. Polym. Environ. 20, 1063-1074(2012).

6) Raychura, A.J.; Jauhari, S.; Prajapati, V.S.; Dholakiya, B.Z. Synthesis and performance evaluation of vegetable oil based wood finish polyurethane coating. Bioresource Technol. Rep. 3, 388-394(2018).

7) McKenzie, W.; Karpovich, M.H. The frictional behaviour of wood. Wood Sci. Technol. 2, 139-152(1968).

8) Klamecki, B.E. Friction mechanisms in wood cutting. Wood Sci. Technol. 10, 209-214 (1976).

9) Aita, Y.; Asanuma, N.; Takahashi, A.; Mayama, H.; Nonomura, Y. Nonlinear friction dynamics on polymer surface under accelerated movement. AIP Adv. 7, 045005 (2017).

10) Aita, Y.; Asanuma, N.; Takahashi, A.; Mayama, H.; Nonomura, Y. Sliding profile and energy at static friction between polymer surface. Chem. Lett. 47, 767-769 (2018).

11) Asanuma, N.; Aita, Y.; Nonomura, Y. Tactile texture of cosmetic sponges and their friction behavior under accelerated movement. J. Oleo Sci. 67, 1117-1122 (2018).

12) Kikegawa, K.; Kuhara, R.; Kwon, J.; Sakamoto, M.; Tsuchiya, R.; Nagatani, N.; Nonomura, Y. Physical ori- gin of a complicated tactile sensation: "Shittori feel". R. Soc. Open Sci. 6, 190039 (2019).

13) Tsuchiya, R.; Kuhara, R.; Kikegawa, K.; Nagatani, N.; Nonomura, Y. Tactile and physical properties of cosmetic powders with a shittori feel. Bull. Chem. Soc. Jpn. 93, 399-405 (2020).

14) Kato, Y.; Kuhara, R.; Sakamoto, M.; Tsuchiya, R.; Nagatani, N.; Nonomura, Y. Recognition mechanism of the "sara-sara feel" of cosmetic powders. J. Oleo Sci. 70, 195-202 (2021).

15) Kuramitsu, K.; Nomura, T.; Nomura, S.; Maeno, T.; Nonomura, Y. Friction evaluation system with a human finger model. Chem. Lett. 42, 284-285(2013).

16) Miyajima, H. Res. Bull. College Experiment Forests Hokkaido Univ. 17, 749-768(1955).

17) Derler, S.; Rotaru, G.M. Stick-slip phenomena in the friction of human skin. Wear 301, 324-329 (2013).

18) Owens, D.K.; Wendt, R.C. Estimation of the surface free energy of polymers. J. Appl. Polymer Sci. 13, 1741-1747 (1969).

19) Brandrup, J.; Immergut, E.H. Polymer Handbook Third Edition, Chapter VI, John Willey \& Sons (1990).

20) Sett, S.; Yan, X.; Barac, G.; Bolton, L.W.; Miljkovic, N. Lubricant-infused surfaces for low-surface-tension fluids: promise versus reality. ACS Appl. Mater. Interfaces 9, 36400-36408 (2017).

21) Nishi, T.; Yamaguchi, T.; Shibata, K.; Hokkirigawa, K. Friction behavior between an artificial skin block and a glass plate under unlubricated and partly/completely water-lubricated conditions. Tribology Int. 163, 107179 (2021).

22) Greenwood, J.A.; Johnson, K.L.; Matsubara, E. A surface roughness parameter in Hertz contact. Wear 100, 47-57(1984).

CC BY-SA 4.0 (Attribution-ShareAlike 4.0 International). This license allows users to share and adapt an article, even commercially, as long as appropriate credit is given and the distribution of derivative works is under the same license as the original. That is, this license lets others copy, distribute, modify and reproduce the Article, provided the original source and Authors are credited under the same license as the original. 